\title{
Gynandropsis gynandra (Capparidaceae) citée pour la première fois comme hôte d'Eurystylus spp. (Hemiptera, Miridae)
}

\author{
par Alain RATNADASS ${ }^{*(1)}$, Younoussou RABO**, Halarou SALHA*, \\ Armand MATOCQ*** \& Ali DOUMMA** \\ * ICRISAT, B. P. 12404, Niamey, Niger <alain.ratnadass@ cirad.fr> \\ ** Université Abdou Moumouni, Faculté d'Agronomie, B.P.10960, Niamey, Niger \\ *** Muséum national d'Histoire naturelle, département Systématique et Evolution, UMR CNRS 7205, \\ C. P. 50 (Entomologie), 57 rue Cuvier, F - 75231 Paris cedex 05 <matocq.armand@wanadoo.fr> \\ (1) Adresse actuelle : CIRAD, UPR HortSys, F - 34398 Montpellier
}

Résumé. - De juillet à octobre 2010 au Niger, nous avons observé en abondance des adultes et tous les stades pré-imaginaux d'Eurystylus bellevoyei, ainsi que quelques adultes d'Eurystylus oldi sur Gynandropsis gynandra (Capparidaceae). Ceci suggère que cette plante est l'hôte préférentiel, jusqu'alors inconnu, d'E. bellevoyei et qu'elle pourrait jouer un rôle-clé dans le cycle biologique d'E. oldi, ravageur majeur du Sorgho en Afrique de l'Ouest.

Abstract. - Gynandropsis gynandra (Capparidaceae), new host plant for Eurystylus spp. (Hemiptera, Miridae). From July to October 2010 in Niger, large numbers of adults and all immature stages of Eurystylus bellevoyei and a few adults of Eurystylus oldi were observed on Gynandropsis gynandra (Capparidaceae). This suggests that this plant is the preferred host, so far unknown, of E. bellevoyei, and that it could play a key-role in the life cycle of E. oldi, a key-pest of Sorghum in West Africa.

Keywords. - Alternative host, Sorghum head bug, Eurystylus oldi, Eurystylus bellevoyei, Niger.

Les punaises Mirides du genre Eurystylus Stål, 1871, revêtent une importance particulière en tant que ravageurs paniculaires du Sorgho [Sorghum bicolor (L.) Moench] en Afrique occidentale et centrale, et dans une moindre mesure du Ricin (Ricinus communis L.) en Afrique australe (STONEDAHL, 1995).

Il est à présent admis (STONEDAHL, 1995) que l'espèce largement dominante sur Sorgho est Eurystylus oldi Poppius, 1912, les autres espèces citées ayant depuis été mises en synonymie avec cette dernière (e.g. E. immaculatus Odhiambo, 1958 : RATNADASS et al., 1994), ou étant le fait d'erreurs d'identification (e.g. E. marginatus Odhiambo, 1958, et E. rufocunealis Poppius, 1911 : GAHUKAR et al., 1989 ; STECK et al., 1989 ; MACFARLANE, 1989). Ces erreurs sont notamment liées au grand polymorphisme de l'espèce, en particulier sur Sorgho (STONEDAHL, 1995 ; SHARMA \& RATNADASS, 2000). L'existence d'une autre espèce occasionnant des dégâts mineurs [E. bellevoyei (Reuter, 1879)] est aussi admise.

C'est notamment le cas au sud du Niger (STECK et al., 1989), où la principale espèce de Miride occasionnant des dégâts au Sorgho a été identifiée comme Eurystylus marginatus, mais STONEDAHL (1995) a entre temps soupçonné qu'il s'agissait d'une erreur d'identification. Au sud du Niger, si E. oldi / E. marginatus représentait 99,5\% de tous les Eurystylus collectés sur Sorgho, une autre espèce était identifiée de façon préliminaire comme E. argenticeps Odhiambo, 1958 (STECK et al., 1989), espèce depuis mise en synonymie avec E. bellevoyei (STONEDAHL, 1995). Cette espèce s'est par ailleurs avérée comme ravageur paniculaire du Sorgho en Inde (SHARMA \& LOPEZ, 1990), où E. bellevoyei a représenté sur Sorgho en 1981 à Patancheru 0,01\% d'une entomofaune de punaises Mirides dominée par Calocoris angustatus Lethierry, 1893. En revanche, c'est aussi une erreur d'identification qui serait à l'origine du signalement par NwANZE (1985) d'E. bellevoyei comme l'espèce la plus dommageable au Sorgho au Burkina Faso (STONEDAHL, 1995). 
La bio-écologie d'E. oldi a été assez largement étudiée (RATNADASS et al., 1994 ; RATNADASS \& BUTLER, 2003), et plusieurs plantes-hôtes alternatives ont été identifiées (RATNADASS et al., 1997 ; AJAYI \& AJIBOYE, 1997 ; MALDÈs \& RATNADASS, 1998). Les seules sur lesquelles tous les stades pré-imaginaux de l'insecte ont été trouvés sont le Sorgho (RATNADASS et al., 1994), le Ricin (RATNADASS et al., 1997), Crotalaria juncea L. (MALDÈs \& RATNADASS, 1998), et le Pois d'angole Cajanus cajan L. (Ratnadass, in litt.).

En revanche, hormis des données sur le cycle de l'espèce sur Sorgho (SHARMA \& LOPEZ, 1990), la bio-écologie d'E. bellevoyei est très peu connue, bien que ce soit l'espèce la plus largement distribuée et considérée comme la plus polyphage (STONEDAHL, 1995).

Le 24 juillet 2010, suite à des observations visuelles préliminaires, nous avons procédé à des échantillonnages par fauchage sur des plantes de Gynandropsis gynandra (L.) Briq. (Capparidaceae) poussant de façon spontanée à Birni N'Konni au Niger $\left(13^{\circ} 47^{\prime} \mathrm{N}, 5^{\circ} 15^{\prime} \mathrm{E}\right)$, puis le 30 juillet avec un D-Vac ${ }^{\circledR}$ [un appareil pneumatique conçu par DIETRICK (1961) pour échantillonner les adultes et larves du Miride Lygus hesperus Knight, 1917, sur Fraisier en Californie], à Sadoré (département de Say, $13^{\circ} 15^{\prime} \mathrm{N}, 2^{\circ} 17^{\prime} \mathrm{E}$ ) où les observations ont été répétées le 10 septembre.

Les résultats, portés au tableau I, montrent que des adultes des deux espèces d'Eurystylus et les cinq stades larvaires d'E. bellevoyei ont été trouvés (fig. 1). L'observation à la loupe binoculaire a par ailleurs révélé la présence d'œufs de Mirides, insérés dans les tissus tendres, notamment les gousses immatures, avec leur operculum faisant saillie à l'extérieur.

Tableau I. - Populations d'Eurystylus spp. relevées sur Gynandropsis gynandra (L.) Briq. au Niger en 2010.

\begin{tabular}{|c|c|c|c|c|c|}
\hline Site et date d'observation & $\begin{array}{c}\text { E. oldi } \\
\text { adultes }\end{array}$ & $\begin{array}{c}\text { E. oldi } \\
\text { larves }\end{array}$ & $\begin{array}{c}\text { E. bellevoyei } \\
\text { adultes }\end{array}$ & $\begin{array}{c}\text { E. bellevoyei } \\
\text { larves (5 stades) }\end{array}$ & $\begin{array}{c}\text { Eurystylus / } \\
\text { total Mirides } \\
(\%)\end{array}$ \\
\hline Birni N'Konni 24.VII.2010 & 2 & 0 & 50 & 57 & $87 \%$ \\
\hline Sadoré 30.VII.2010 & 8 & 0 & 91 & 63 & $93 \%$ \\
\hline Sadoré 10.IX.2010 & 3 & 0 & 14 & 21 & $\begin{array}{c}\text { non } \\
\text { déterminé }\end{array}$ \\
\hline
\end{tabular}

Ainsi, les Eurystylus ont représenté l'essentiel des Mirides sur Gynandropsis gynandra, $E$. bellevoyei étant largement dominant, $E$. oldi n'ayant représenté que respectivement 1,8\% des Eurystylus à Birni N'Konni en juillet, et 4,9\% à Sadoré en juillet et 7,9\% en septembre.

Après la récolte du sorgho, le 19 novembre, une observation qualitative ponctuelle à Sadoré à permis d'identifier E. bellevoyei sur G. gynandra, mais pas E. oldi.

Bien qu'aucun stade pré-imaginal d'E. oldi n'ait été trouvé sur G. gynandra, c'est la première fois qu'E. oldi est signalé sur une plante-hôte aussi tôt en saison, i. e. bien avant la floraison du sorgho, les précédentes observations ayant été effectuées au plus tôt fin aoûtdébut septembre au Mali (RATNADASS \& BUTLER, 2003). A ce titre, cette découverte contribuera à "boucler" le cycle complet de l'espèce.

Concernant $E$. bellevoyei, c'est la première fois que les cinq stades larvaires de l'espèce ont été trouvés sur ce qui semble être son hôte hautement préférentiel, même si l'espèce a déjà été signalée sur d'autres Capparidaceae (Cleome sp. et Dipterygium sp.) (STONEDAHL, 1995), sachant que G. gynandra a aussi été considéré comme appartenant au genre Cleome. Eurystylus bellevoyei est associé aux Chenopodiaceae en Europe, où l'espèce a été signalée sur plusieurs îles (Sicile, Pantelleria, Chypre, Canaries), ainsi que sur le continent en Catalogne (CARAPEZZA, 1998).

Plante indigène africaine, Gynandropsis gynandra est un important légume-feuille en Afrique occidentale, y compris au Niger (CHWEYA \& MNZAVA, 1997), bien qu'il y revête une importance moindre qu'en Afrique orientale et australe où il fait même l'objet de programmes 


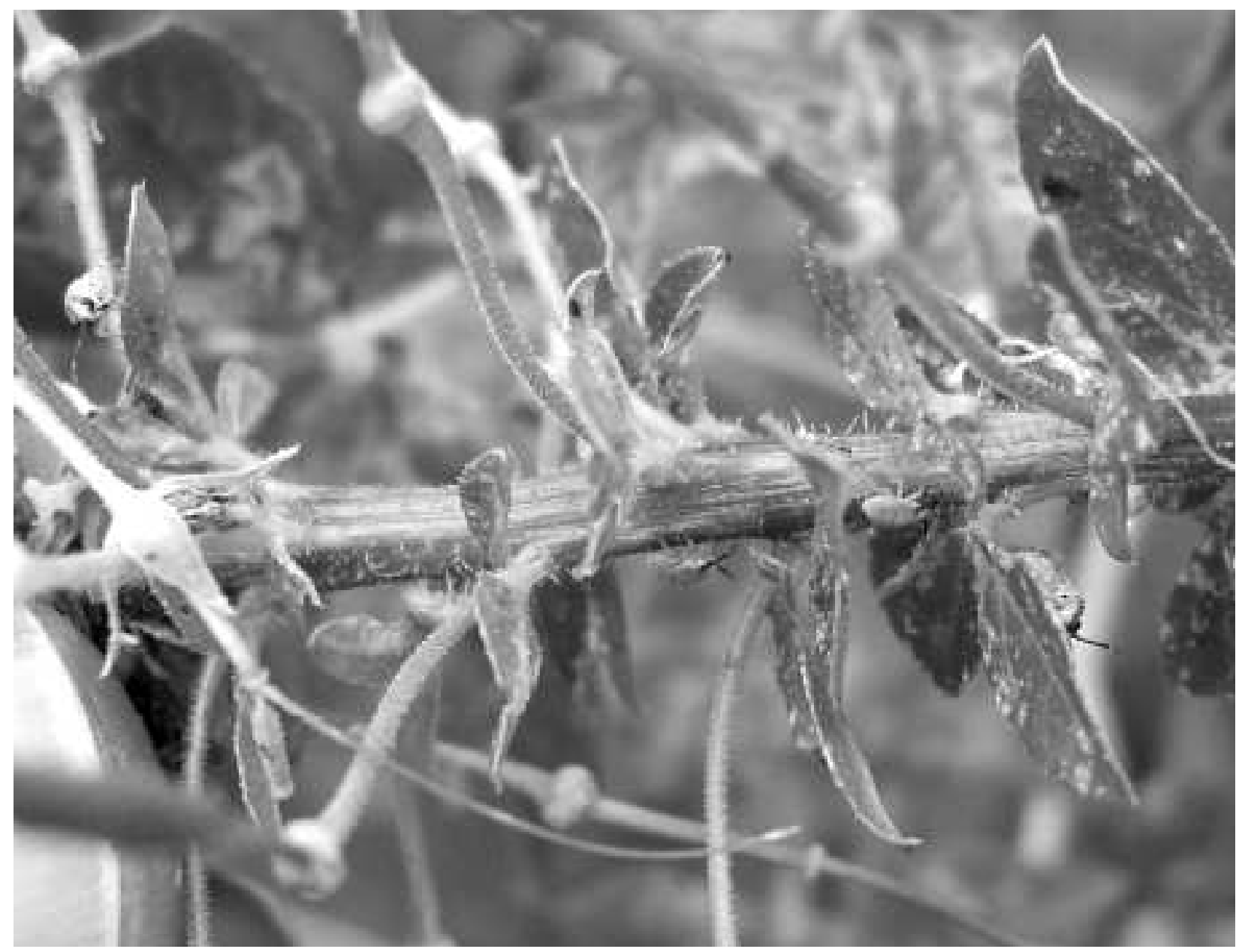

Fig. 1. - Adultes et larves d'Eurystylus bellevoyei sur Gynandropsis gynandra (Birni N'Konni, Niger, Juillet 2010).

d'amélioration variétale (AVRDC à Arusha en Tanzanie, Zambia Seed Company), notamment en Zambie et au Kenya où ses semences sont commercialisées. Bien que peu attaqué par les Insectes, ce qui peut être mis en relation avec les propriétés insecticides ou insectifuges de certains de ses extraits, plusieurs Punaises Pentatomidae figurent dans la liste des ses Insectes ravageurs (genres Nezara, Acrosternum, Agonoscelis et Bagrada), mais aucun Miride n'avait à ce jour été signalé.

\section{AUTEURS CITÉS}

AJAYI A. \& AJIBOYE T. O., 1997. - Non crop host plants of the sorghum head bug, Eurystylus oldi in west Africa. International Sorghum and Millets Newsletter, $38: 81-82$.

CARAPEZZA A., 1998. - New species and new records of Heteroptera from Cyprus (Insecta). Atti dell'Accademia Roveretana degli Agiati, (VII) 8 B : 29-40.

ChweYA J. A. \& MnZAVA N. A., 1997. - Cat's whiskers. Cleome gynandra L. Promoting the conservation and use of underutilized and neglected crops. 11. Institute of Plant Genetics and Crop Plant Research, Gatersleben/International Plant Genetic Resources Institute, Rome, Italy. 54 p.

DIETRICK E. J., 1961. - An improved backpack motor fan for suction sampling of insect populations. Journal of Economic Entomology, 54 : 394-395.

GAHUKAR R. T., DOUMBia Y. O., BAL A. B. \& BHATNAGAR V. S., 1989. - Eurystylus marginatus Odh., a new pest of sorghum in the Sahel. Tropical Pest Management, 35 : 212-213.

MACFARLANE J., 1989. - The hemipterous insects and spiders of sorghum panicles in northern Nigeria. Insect Science and its Application, 10 : 277-284.

MALDÈS J. M. \& RATNADASS A., 1998. - Crotalaria juncea (Fabaceae) citée pour la première fois comme hôte alternatif d'Eurystylus oldi important ravageur africain du Sorgho et du Ricin (Hem., Miridae). Bulletin de la Société entomologique de France, 103 (3) : 272. 
NWANZE K. F., 1985. - Sorghum insect pests in West Africa (p. 37-43). In : Proceedings of the International Sorghum Entomology Workshop, 15-21 July, 1984, Texas A\&M University, College Station, Texas, USA. Patancheru, AP 502 324, India: ICRISAT.

RATNADASS A. \& BUTLER D. R., 2003. - Abundance of sorghum panicle-feeding bugs (Hemiptera: Miridae) in Mali and empirical relationships with weather. Insect Science and its Application, 3 : 239-250.

Ratnadass A., Cissé B., Diarra D. \& SAngaré M. L., 1997. - Indigenous host plants of sorghum head-bug (Heteroptera: Miridae) in Mali. African Entomology, 5 (1) : 158-160.

RATNADASS A., CissÉ B. \& MAllÉ K., 1994. - Note on the biology and immature stages of West African sorghum head bugs Eurystylus immaculatus and Creontiades pallidus (Heteroptera: Miridae). Bulletin of Entomological Research, 84 : 383-388.

SHARMA H. C. \& LOPEZ V. F., 1990. - Biology and population dynamics of sorghum head bugs (Hemiptera: Miridae). Crop Protection, 9 : 164-173.

SHARMA H. C. \& RATNADASS A., 2000. - Colour variation in the African sorghum head bug. International Sorghum and Millets Newsletter, 41 : 42-43.

Steck G., TeEtes G. L. \& MAÏGA S. D., 1989. - Species composition and injury to sorghum by panicle-feeding bugs in Niger. Insect Science and its Application, 10 : 199-217.

STONEDAHL G. M., 1995. - Taxonomy of African Eurystylus (Heteroptera: Miridae), with a review of their status as pests of sorghum. Bulletin of Entomological Research, 85 : 135-136.

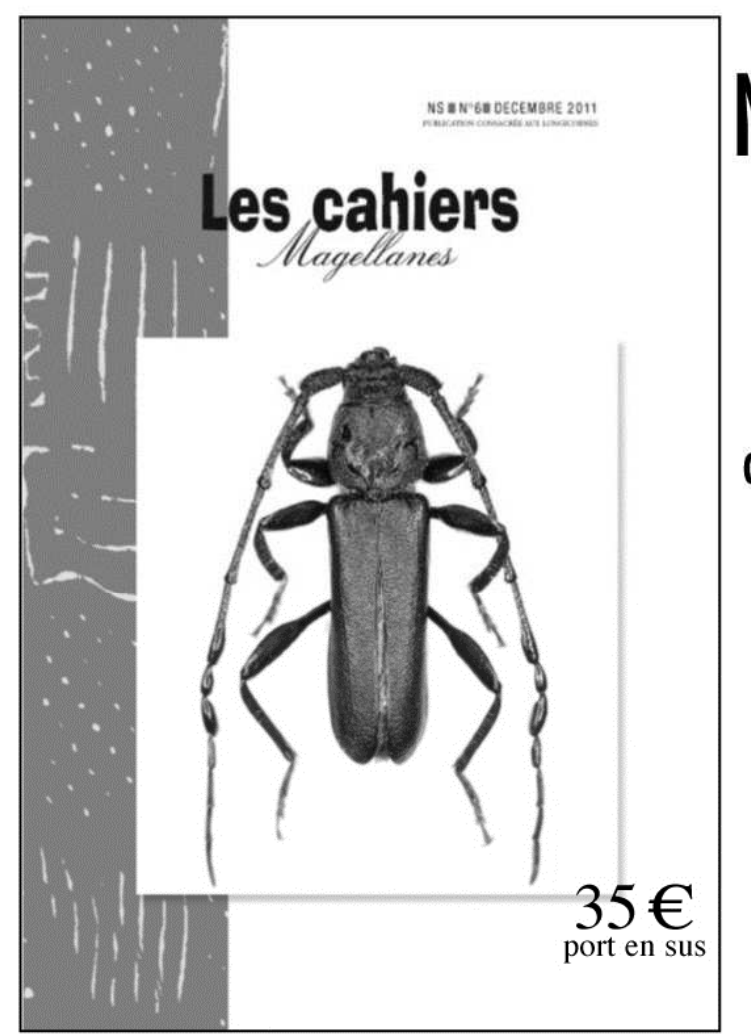

\title{
NOUVEAUTÉ MAGELLANES Décembre 2011
}

Nouveau Cahiers Magellanes $n^{\circ} 6$ collection dédiée aux Longicornes du monde

\author{
125 pages, 27 Planches couleur,
}

\section{Ce cahier traite de la faune d'Afrique, d'Amérique du Sud et d'Asie du Sud-Est}

Vente à l'unité ou par abonnement

Renseignements ou commande par courrier à : Magellanes 10, rue de la Gare 78570 Andrésy France, ou par email : ciiroux@wanadoo.fr 